\title{
Magnetic field amplification by turbulent dynamo in relativistic collisionless shocks
}

\author{
Sara Tomita ${ }^{a, b, *}$ and Yutaka Ohira ${ }^{c}$ \\ ${ }^{a}$ Frontier Research Institute for Interdisciplinary Sciences, Tohoku University, \\ Sendai, 980-8578, Japan \\ ${ }^{b}$ Astronomical Institute, Graduate School of Science, Tohoku University, \\ Sendai, 980-8578, Japan \\ ${ }^{c}$ Department of Earth and Planetary Science, The University of Tokyo, \\ 7-3-1 Hongo, Bunkyo-ku, Tokyo 113-0033, Japan \\ E-mail: tomisara@astr.tohoku.ac.jp, y.ohira@eps.s.u-tokyo.ac.jp
}

Cosmic rays are thought to be efficiently produced in collisionless shocks in high-energy astrophysical sources, where cosmic rays are diffusively scattered by magnetic fluctuations. The magnetic field near the shock decides the maximum energy of cosmic rays accelerated in the shock and the emission by the accelerated particles. However, the magnetic field strength and structure around the shock are not understood yet. Recent magnetohydrodynamics (MHD) simulations of shocks propagating into inhomogeneous media show that the ambient magnetic field is amplified by a turbulent dynamo in the downstream region. According to these simulations, the turbulent dynamo always works as long as the magnetic energy is smaller than the kinetic energy of the downstream turbulence. However, the shocks formed in astrophysical phenomena are often driven by collisionless plasma, where non-thermal particles are generated, so that it is unknown whether or not the MHD approximation is applicable to the downstream flow. In particular, for shocks in gamma-ray bursts, the size of density fluctuations has to be about ten times the gyroradius of the thermal protons to amplify the magnetic field by the downstream turbulence. We perform particle-in-cell simulations of relativistic collisionless shocks propagating into a pair plasma with a density clump whose size is ten times the gyroradius of downstream thermal plasmas. We found that the magnetic field amplification does not work if the amplitude of the upstream density fluctuation is below a critical value.

$37^{\text {th }}$ International Cosmic Ray Conference (ICRC 2021)

July 12th - 23rd, 2021

Online - Berlin, Germany

\footnotetext{
*Presenter
} 


\section{Introduction}

Collisionless shocks are thought to be important in the energy dissipation in high-energy astrophysical phenomena. In the collisionless shocks, non-thermal particles and strong magnetic fields are generated, which produce high-energy photons through synchrotron radiation. The maximum energy of accelerated particles in the shock and the spectrum of synchrotron emission in the source depend strongly on magnetic fields around the shock. However, it's highly uncertain. Main problems are as follows. First, we have not understood the amplification mechanism of post-shock magnetic fields. A simple shock compression cannot explain the magnetic field strength suggested by observations. In addition, particle acceleration does not efficiently work at the shock if the interstellar magnetic is just compressed by the shock. Second, in many cases, we have no information about the upstream region. Information about surroundings must tell us about the origin of high-energy astrophysical phenomena. Therefore, it is important to understand the mechanism of magnetic field amplification in the shocks, which could make it possible for us to search the environment of astrophysical object. So far, two mechanisms of magnetic field amplification in collisionless shocks have been investigated. One is kinetic plasma instabilities, and the other is turbulent dynamo. Kinetic plasma instabilities generate small-scale magnetic fields which are important for the particle acceleration[1, 2, 7, 11-16]. On the other hand, the magneto hydrodynamic (MHD) turbulence amplifies the magnetic field $[3-5,8,10,18]$, so that synchrotron radiation efficiently generates high-energy photons. Therefore, we need both of them to explain high-energy astrophysical observations.

In our study, we focus on the turbulent dynamo in relativistic collisionless shocks. Previous MHD simulations showed that a large-scale magnetic field is amplified by the turbulence in the downstream region. The turbulent dynamo is expected to work as long as the kinetic energy of the downstream turbulence is larger than the magnetic energy[9]. However, the magnetic field generation by the kinetic plasma instabilities and particle accelerations cannot be solved in the MHD simulation. This means that the MHD simulations cannot solve the magnetic field amplification concerning accelerated high-energy particles. High-energy astrophysical shocks are usually collisionless shocks, and non-thermal particles are generated as mentioned above. We expect that particle diffusion have an important role in the collisionless shock. The mean free path of the high-energy particles becomes very large. It is unclear whether or not density fluctuations in the collisionless shock are dissipated by the particle diffusion in the downstream region. In order for MHD approximation to be applicable in the downstream regions of collisonless shocks, the spatial scale of the density fluctuation must be larger than the gyro radius, but we do not know how large size is needed. Not only the lengthscale but also the amplitude of the density fluctuations could be an important parameter.

To understand whether the turbulent dynamo works in relativistic collisionless shocks or not, we perform Particle-In-Cell (PIC) simulation for a relativistic collisionless shock propagating to a nonuniform medium. We find that if the upstream density perturbation is small, that is, $\delta n / n<0.5$, the turbulent dynamo amplification does not work in the downstream region of the relativistic collisionless shocks. 


\section{Simulation Setup}

We performed two-dimensional PIC simulations of a relativistic collisionless shock using the open source code, pCANS. The simulation box is in the $x-y$ plane where the periodic boundary condition is imposed in the y-direction. The simulation box size is $L_{x}=3120 \mathrm{c} / \omega_{\mathrm{pe}}$ and $L_{y}=$ $1200 c / \omega_{\text {pe }}$. The plasma skin-depth $c / \omega_{\text {pe }}$ is given by

$$
\frac{c}{\omega_{\mathrm{pe}}}=\sqrt{\frac{\Gamma m_{\mathrm{e}} c^{2}}{4 \pi n_{0} e^{2}},}
$$

where $n_{0}$ and $\Gamma$ are the upstream mean number density and bulk Lorentz factor measured in the downstream rest frame, and the constants $c, e, m_{\mathrm{e}}$ are speed of light, particle charge, particle mass, respectively. The cell size and time step are $\Delta x=\Delta y=0.1 \mathrm{c} / \omega_{\mathrm{pe}}$ and $\Delta t=0.1 \omega_{\mathrm{pe}}^{-1}$, respectively.

The magnetized upstream electron-positron plasma with $\Gamma=10$ and thermal velocity of $0.18 c$ are injected at the right boundary and reflected at the left boundary. The reflected particles interact with the incoming particles through electromagnetic fields, so that a shock is formed and propagates toward the injection wall. The simulation frame is the downstream rest frame, that is, the downstream mean velocity is zero. We use 40 particles per cell per species in the upstream region. We set one density clump in the upstream region for simplification. The density distribution of the clump is set to be

$$
n(x, y)=n_{0}\left[1+\delta\left(1+\cos \left(\pi r / r_{\mathrm{c}}\right)\right)\right],
$$

where $n_{0}$ is the upstream mean number density in the simulation frame, $r_{\mathrm{c}}$ is the clump size which is defined by half width at half maximum amplitude $\delta$. In this simulation, the clump size is set to be $r_{\mathrm{c}}=300 \mathrm{c} / \omega_{\mathrm{pe}}$. The upstream magnetic field is in the y-direction. The magnetic field strength is characterized by the sigma parameter defined by $\sigma_{\mathrm{e}}=B_{0}^{2} /\left(4 \pi \Gamma n_{0} m_{\mathrm{e}} c^{2}\right)$, where $B_{0}$ is the amplitude of upstream magnetic field in the simulation frame. Model parameters are summarized in table 1. For early phase of afterglows of Gamma-ray bursts, in order to explain the expected magnetic field, the turbulent dynamo model requires that the size of the upstream density clump is less than $10^{17}$ $\mathrm{cm}$ in the upstream rest frame[10]. If the upstream magnetic field is $3 \mu \mathrm{G}$ in the upstream rest frame, the clump size becomes about 10 times gyroradius of the downstream thermal protons. In the downstream region of relativistic shocks, the electron energy is expected to be almost the same as the proton energy[6], so that the ratio of the clump size to the gyroradius of electrons is also about 10. For the case 1 and 4, the size ratio is set to be about 10 .

\begin{tabular}{cccc}
\hline Model & $\delta$ & $\sigma_{\mathrm{e}}$ & $2 r_{\mathrm{c}} / r_{\mathrm{ge}}$ \\
\hline \hline case 1 & 0.5 & $10^{-3}$ & 9.5 \\
case 2 & 0.5 & $10^{-4}$ & 3.0 \\
case 3 & 0.5 & $10^{-5}$ & 0.9 \\
case 4 & 10.0 & $10^{-3}$ & 9.5 \\
\hline
\end{tabular}

Table 1: Model List

We also use the relativistic MHD code "Athena++" [17] to simulate the relativistic shock propagating to an inhomogeneous medium. We compare the results for the PIC and MHD simulation. 

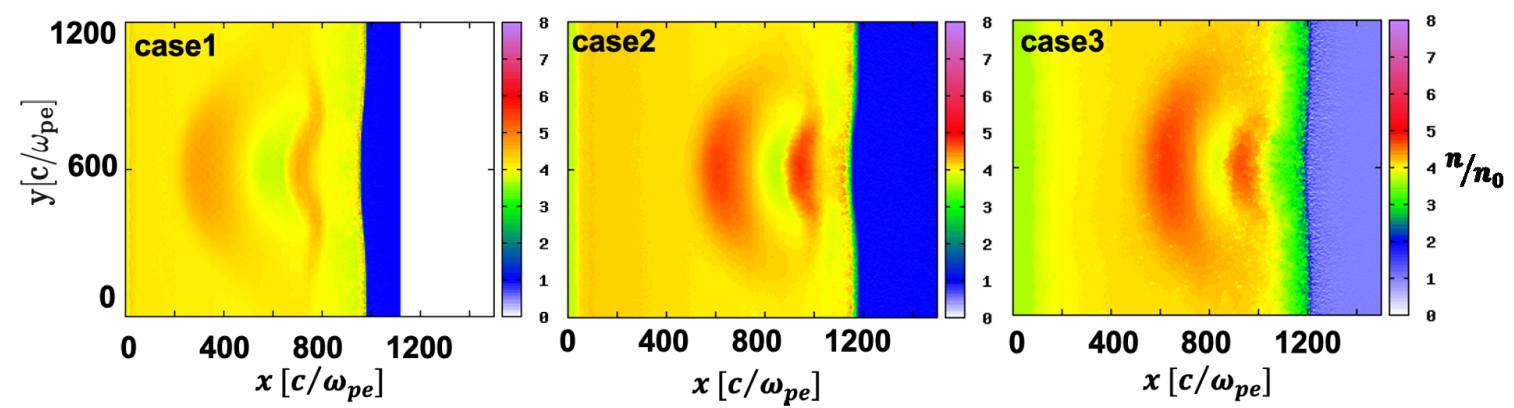

Figure 1: Result of PIC for density distributions in the shock downstream region at $t=800 \omega_{\mathrm{pe}}^{-1}$ after the clump is injected to the shock. The case 1, 2, and 3 are results for $\sigma=10^{-3}, \sigma=10^{-4}$, and $\sigma=10^{-5}$, respectively. The blue region is the upstream region.

The initial model parameters such as the clump size and amplitude, bulk Lorentz factor, pressure, and magnetic field strength and direction in the upstream regions of the shock are set to be the same as that in PIC simulation.

\section{Results}

We investigate the evolution of the density clump and the magnetic field amplification by the downstream turbulence that is driven by the upstream density clump. Figure 1 shows the density distribution for the case 1,2, and 3 at $t=800 \omega_{\mathrm{pe}}^{-1}$ after the clump is injected to the shock front. The blue and yellow regions are the upstream and downstream region, respectively. In our simulation, the injection boundary moves to the upstream region at every a few time steps, there is no particle in the white regions yet. After the clump passes through the shock front, particles diffusively escape from the clump along the magnetic field line. The magnetic field direction is the $y$-direction. In addition, the sound wave is propagating to the left direction.

In Figure 2, we compare the results for the PIC simulation to that for MHD simulation. The left and right panels in the Figure 2 show the downstream density distribution for the PIC and MHD simulation, respectively. For the MHD simulation, the high-density clump is not dissipated by the particle diffusion, but for the PIC simulation, particles in the high-density clump diffuse along the magnetic field line. Therefore, in this PIC simulation, we cannot expect the magnetic field amplification by the turbulent dynamo because the turbulence is not driven by the high-density clump.

Finally, we show the result of $\delta$-dependence in Figure 3. The upstream magnetic field strength is fixed at $\sigma_{\mathrm{e}}=10^{-3}$ in both cases. The left and right panels in Figure 3 show the density distributions for the case of 4 and 1, respectively. For the higher-density clump, the eddy like structure around the clump is observed in the downstream region because the particle diffusion is suppressed. As a result, the post-shock magnetic field can be amplified by the turbulent dynamo. Therefore, a high-density clump is needed to amplify the magnetic field by the turbulent dynamo. This would be because the thermal velocity of particles in the shocked clump is lower than that for the case of smaller-density amplitude. Then, the magnetic field line is stretched by the shear flow before particles in clump escape from the clump region. Once the magnetic field line is strongly stretched, 


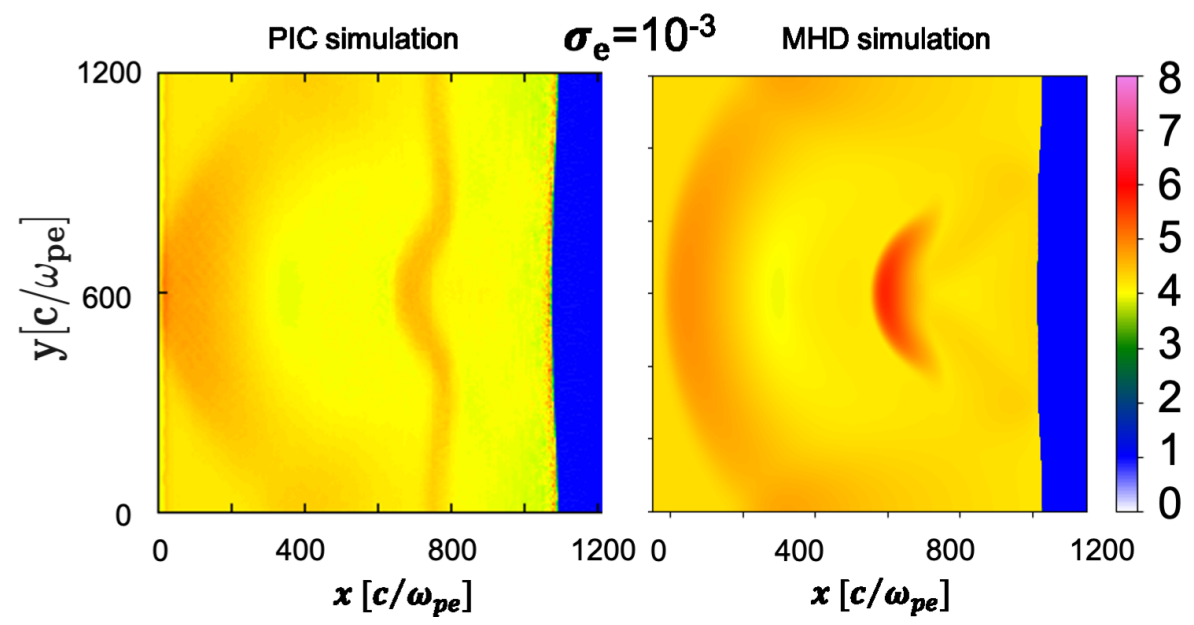

Figure 2: Density distributions in the shock downstream region at $t=1150 \omega_{\mathrm{pe}}^{-1}$ after the clump is injected to the shock. The left and right figures are the result for PIC simulation and that for MHD simulation, respectively. Both of them are the case of $\sigma=10^{-3}$.
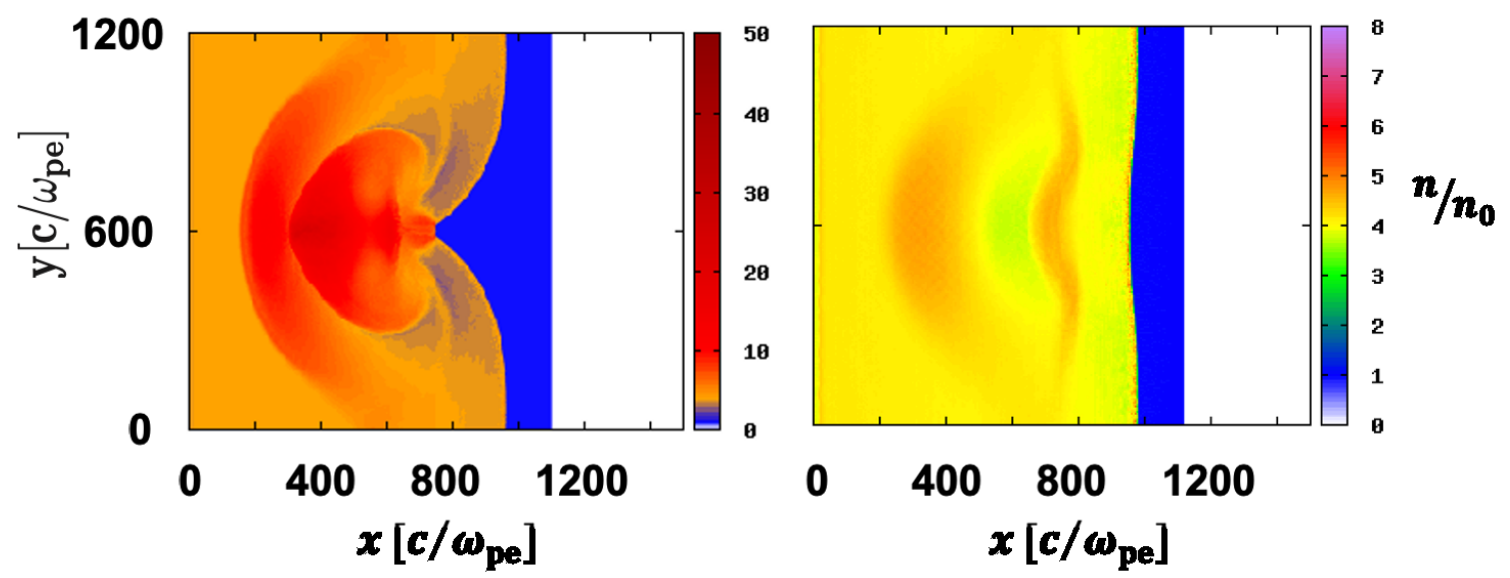

Figure 3: Density distributions in the shock downstream region at $t=800 \omega_{\mathrm{pe}}^{-1}$ after the clump is injected to the shock. The case 1 and 4 are $\delta=0.5$ and $\delta=10.0$, respectively. Both of them are the case of $\sigma=10^{-3}$.

particles cannot escape along the magnetic field line. Therefore, a high-density clump is needed to amplify the magnetic field by the turbulent dynamo. The parameter dependence on the downstream turbulence is being investigated (Tomita et al. 2021, in preparation).

\section{Summary}

In order to find the upstream environment required from the turbulent dynamo amplification in collisionless shocks, we performed PIC simulations of relativistic collisionless shocks propagating to an inhomogeneous medium. We found that the density fluctuation with small amplitude such an interstellar turbulence cannot drive a strong turbulent dynamo amplification even if the clump size is 10 times the gyroradius of thermal particles. Thus, in order for the magnetic field to be amplified, the amplitude of the density clump has to be sufficiently large. 


\section{Acknowledgemnts}

We thank K. Toma, K. Tomida, S. Kimura, A. Kuwata, and R. Kuze for useful comments. The software used in this work was in part developed in pCANS at Chiba University. Numerical computations were carried out on Cray X50 at Center for Computational Astrophysics, National Astronomical Observatory of Japan. This work is supported by JSPS KAKENHI grant numbers 19H01893 (YO).

\section{References}

[1] Bell, A. R., Turbulent amplification of magnetic field and diffusive shock acceleration of cosmic rays, MNRAS, 353, 550, 2004

[2] Drury, L. O., \& Downes, T. P., Turbulent magnetic field amplification driven by cosmic ray pressure gradients, MNRAS, 427, 2308, 2012

[3] Giacalone, J., \& Jokipii, J. R., Large-scale turbulence, shocks, and charged-particle acceleration, AIPC, 932, 243, 2007

[4] Inoue, T., Yamazaki, R., \& Inutsuka, S.-. ichiro., Turbulence and Magnetic Field Amplification in Supernova Remnants: Interactions Between a Strong Shock Wave and Multiphase Interstellar Medium, ApJ, 695, 825, 2009

[5] Inoue, T., Asano, K., \& Ioka, K., Three-dimentinal simulations of magnetohydrodynamic turbulence behind relativistic shock waves and their implications for gamma-ray bursts, ApJ, $734,77,2011$

[6] Kumar, R., Eichler, D., \& Gedalin, M., Electron Heating in a Relativistic, Weibel-unstable Plasma, ApJ, 806, 165, 2015

[7] Lucek, S. G., \& Bell, A. R., Non-linear amplification of a magnetic field driven by cosmic ray streaming, MNRAS, 314, 65, 2000

[8] Mizuno, Y., Pohl, M., Niemiec, J., et al., Magnetic field amplification and saturation in turbulence behind a relativistic shock, ApJ, 726, 62, 2011

[9] Sano, T., Inoue, T., \& Nishihara, K., Critical Magnetic Field Strength for Suppression of the Richtmyer-Meshkov Instability in Plasmas, PRL, 111, 205001, 2013

[10] Sironi, L., \& Goodman, J., Production of Magnetic Energy by Macroscopic Turbulence in GRB Afterglows, ApJ, 671,1858, 2007

[11] Sironi, L., Spitokovsky, A., \& Arons, J., The maximum energy of accelerated particles in relativistic collisionless shocks, ApJ, 771, 54, 2013

[12] Spitkovsky, A., On the structure of relativistic collisionless shocks in electron-Ion plasmas, ApJ, 673, L39, 2008 
[13] Spitkovsky, A., Particle acceleration in relativistic collisionless shocks: Fermi process at last?, ApJ, 682, L5-L9, 2008

[14] Tomita, S., \& Ohira, Y., Weibel Instability Driven by Spatially Anisotropic Density Structures, ApJ, 825, 103, 2016

[15] Tomita, S., Ohira, Y., \& Yamazaki, R., Weibel-mediated Shocks Propagating into Inhomogeneous Electron-Positron Plasmas, ApJ, 886, 54, 2019

[16] Weibel, E. S., Spontaneously growing transverse waves in a plasma due to an anisotropic velocity distribution, $P R L, 2,83-84,1959$

[17] White, C. J., Stone, J. M., \& Gammie, C. F., An Extension of the Athena++ Code Framework for GRMHD Based on Advanced Riemann Solvers and Staggered-mesh Constrained Transport, ApJS, 225, 22, 2016

[18] Zhang, W., MacFadyen, A., \& Wang, P., Three-Dimensional Relativistic Magnetohydrodynamic Simulations of the Kelvin-Helmholtz Instability: Magnetic Field Amplification by a Turbulent Dynamo, ApJL, 692, L40, 2009 